\title{
Activation of Bcl-2-Associated Death Protein and Counter- Response of Akt within Cell Populations during Seizure-Induced Neuronal Death
}

\author{
David C. Henshall, ${ }^{1}$ Tomohiro Araki, ${ }^{1,3}$ Clara K. Schindler, ${ }^{1}$ Jing-Quan Lan, ${ }^{1}$ Kenneth L. Tiekoter, ${ }^{2}$ Waro Taki, ${ }^{3}$ \\ and Roger P. Simon ${ }^{1}$
}

${ }^{1}$ Robert S. Dow Neurobiology Laboratories and 2Department of Microimaging, Legacy Research, Portland, Oregon

97232, and '3epartment of Neurosurgery, Mie University School of Medicine, Tsu, Mie 541-8507, Japan

Bcl-2 family gene products are critical to the integration of cell death stimuli that target the mitochondrion. Proapoptotic BAD (Bcl-2-associated death protein) has been shown to dissociate from its sequestered site with the molecular chaperone protein 14-3-3 and displace proapoptotic BAX (Bcl-2-associated $X$ protein) from antiapoptotic BCL-XI. BAX subsequently translocates to the mitochondrion and induces cytochrome $c$ release and caspase activation. Herein we report the response of the key members of this proposed pathway after seizures. Seizures evoked by microinjection of kainic acid into the amygdala of the rat induced unilateral CA3 pyramidal neuron death with features of apoptosis. In control hippocampus and cortex, BAD was found constitutively bound to 14-3-3, whereas BCL-XI bound BAX. Within damaged hippocampus, seizures induced the dissociation of BAD from 14-3-3 and the subsequent dimerization of $B A D$ with $B C L-X I$ as determined by immunoprecipitation and

The mitochondrion is a critical site for the initiation and/or reinforcement of cell death pathways (Kroemer and Reed, 2000; Mattson, 2000). The Bcl-2 family comprises proapoptotic and antiapoptotic proteins implicated in the processing and integration of death stimuli targeted at the mitochondrion (Adams and Cory, 1998; Reed, 1998). BAX (Bcl-2-associated X protein) is integral to triggering release of cytochrome $c$, a critical factor in the initiation of cell death pathways originating from the mitochondrion (Korsmeyer et al., 2000; von Ahsen et al., 2000; Wei et al., 2001). Such BAX activation may be initiated or potentiated by another death agonist, BAD (Bcl-2-associated death protein) (Yang et al., 1995). Through phosphorylation of one or more serine residues ( $\mathrm{Z}$ ha et al., 1996; Lizcano et al., 2000; Tan et al., 2000), BAD resides in an inactive complex with the molecular chaperone 14-3-3 (Zha et al., 1996; Datta et al., 2000; Tan et al., 2000). Akt (protein kinase B) is known to phosphorylate BAD at the $\operatorname{Ser}^{136}$ residue that is critical for sequestration to 14-3-3 (Lizcano et al., 2000; Tan et al., 2000; Masters et al., 2001). In turn, Akt activity is positively regulated by phosphorylation on residues $\mathrm{Thr}^{308}$ and $\mathrm{Ser}^{473}$ downstream of phosphatidylinositol 3 (PI3) kinase (Burgering and Coffer, 1995; Datta et al., 1997;

\footnotetext{
Received April 4, 2002; revised July 17, 2002; accepted July 22, 2002.

This work was supported by National Institutes of Health Grants NS39016 (D.C.H., R.P.S.) and NS41935 (D.C.H.).

Correspondence should be addressed to Dr. David C. Henshall, Robert S. Dow Neurobiology Laboratories, Legacy Research, 1225 Northeast Second Avenue, Portland, OR 97232. E-mail: dhenshall@downeurobiology.org.

Copyright (C) 2002 Society for Neuroscience $0270-6474 / 02 / 228458-08 \$ 15.00 / 0$
}

immunohistochemical colocalization. 14-3-3 was found to translocate to the nucleus of degenerating neurons, whereas BAX accumulated at mitochondrial membranes. In contrast, the primarily uninjured cortex exhibited increased phosphorylation of Akt (protein kinase B), which may phosphorylate and inhibit $B A D$, and no altered binding of BAD to BCL-XI. Finally, administration of an inhibitor of phosphatidylinositol 3-kinase (LY294002), thought to be an upstream activator of Akt, exacerbated cortical apoptosis after seizures. These data suggest that seizures elicit divergent cell death and survival responses within neuronal populations and that the BAD cell death pathway may perform an instigator or reinforcement role in seizureinduced neuronal death.

Key words: epilepsy; apoptosis; necrosis; BCL-2; BAX; mitochondria; caspase

Dudek et al., 1997). This inhibitory effect of Akt on BAD has been supported by the demonstration that a PI3 kinase inhibitor, LY294002, exacerbated brain injury after cerebral ischemia (Noshita et al., 2001). After apoptotic stimuli, such as calcineurininduced dephosphorylation (Wang et al., 1999), BAD is released from 14-3-3 and subsequently dimerizes with the antiapoptotic protein BCL-X1 (Yang et al., 1995; Zha et al., 1996). Because BCL-X1 resides constitutively bound to BAX, BAD displaces and releases BAX from BCL-X1 (Yang et al., 1995). Thereafter, BAX translocates to the mitochondrion in which it promotes release of cytochrome $c$, formation of the apoptosome, and activation of the caspase cascade (Li et al., 1997; Zou et al., 1997; Bratton et al., 2001). A schematic of this pathway is shown in Figure $1 A$.

Studies have determined that seizures induce a mixed pattern of cell death that includes features consistent with both apoptosis and necrosis (Pollard et al., 1994; Sloviter et al., 1996; Bengzon et al., 1997; Fujikawa et al., 2000a,b). Activation of mitochondrion-linked, apoptosis-like signaling pathways has been described after seizures, including early cytochrome $c$ redistribution and activation of caspase-9 and caspase-3 (Viswanath et al., 2000; Henshall et al., 2000a, 2001a). The trigger for cytochrome $c$ release after seizures is unknown, but involvement of proapoptotic BID (BH3-interacting domain death agonist) is suggested by the demonstration of caspase- 8 activation and BID truncation after seizures (Henshall et al., 2001b). However, the role of BAD in this cell death pathway has not been addressed. To better define the mechanisms underlying initiation of seizure-induced cell death, we examined the response of the BAD signaling pathway after seizures. 


\section{MATERIALS AND METHODS}

Seizure model. All animal procedures were performed in a facility accredited by the Association for Assessment and Accreditation of Laboratory Animal Care in accordance with protocols approved by the Legacy Institutional Animal Care and Use Committee and the principles outlined in the National Institute of Health Guide for the Care and Use of Laboratory Animals. Studies were performed as described previously (Henshall et al., 2000c) with some modifications. Seizures were focally evoked in adult male Sprague Dawley rats (280-350 gm) by unilateral stereotaxic microinjection of kainic acid (KA) (Sigma, St. Louis, MO) into the basolateral amygdala nucleus. After anesthesia, intubation, and vein catheterization, animals were placed in a stereotaxic frame. Recording electrodes (Plastics One, Roanoke, VA) were then affixed to the skull bitemporally over the hippocampi and a third across frontal cortex to record EEG (Grass Instruments model 8-16). A craniectomy was also performed for placement of the injection cannula. The animal was then removed from the frame, anesthesia was discontinued, EEG recordings were commenced, and a 31 gauge internal cannula (Plastics One) was then inserted into the lumen of the guide to inject KA $(0.1 \mu \mathrm{g}$ in $0.5 \mu \mathrm{l}$ of saline vehicle) into the amygdala. Nonseizure control animals underwent the same surgical procedure but received intra-amygdala vehicle injection. The EEG was monitored continuously until diazepam (30 $\mathrm{mg} / \mathrm{kg}$, i.v.) was administered to terminate seizures after $40 \mathrm{~min}$. The EEG was further monitored for $\leq 1 \mathrm{hr}$ to ensure seizure cessation.

In vivo modulation of $B A D$ pathway. To examine the effect of manipulating the BAD cell death pathway, additional rats received the calcineurin inhibitor FK506 (1 mg/kg, i.v.; a gift from Dr. T. Andoh, Legacy Research, Portland, OR) or vehicle ( $10 \%$ ethanol in saline containing $400 \mathrm{mg} / \mathrm{kg}$ polyoxyl 60 hydrogenated castor oil) $10 \mathrm{~min}$ before KA or vehicle injection. To examine the contribution of the Akt pathway, additional rats received the PI3 kinase inhibitor LY294002 (50 nmol; Cell Signaling Technology, Beverly, MA) or vehicle (dimethylsulfoxide) $10 \mathrm{~min}$ before and $1 \mathrm{hr}$ after KA or vehicle injection. Brains were obtained $72 \mathrm{hr}$ after diazepam and processed for histopathology. The duration of polyspike paroxysmal discharges was quantified for all animals in these studies to ensure that treatment groups received equivalent durations of injury-producing seizures as described previously (Henshall et al., 2001b).

Western blotting. Animals were killed 0, 4, 8, 24, or $72 \mathrm{hr}$ after administration of diazepam in seizure animals or after 4 or $24 \mathrm{hr}$ in nonseizure controls, and ipsilateral and contralateral hippocampus and piriform cortex were obtained. Pooled ( $n=2-3$ per group) brain samples were homogenized and lysed in buffer containing $1 \%$ Nonidet P-40 (NP-40) and the following protease inhibitors (in $\mu \mathrm{g} / \mathrm{ml}$ ): 100 phenylmethylsulfonyl fluoride, 1 leupeptin, 1 pepstatin, and 1 aprotinin. Lysates were cleared by centrifugation, and protein concentration was determined using Bradford reagent spectrophotometrically at $A^{595} \mathrm{~nm}$. Samples $(50 \mu \mathrm{g})$ were boiled in gel-loading buffer and then separated on $12 \%$ SDS-PAGE gels. Proteins were transferred to polyvinylidene difluoride membranes (Bio-Rad, Hercules, CA) and then incubated with the following antibodies: anti-Akt, anti-phosphoAkt ${ }^{308,473}$, and anti-BAD (polyclonal; Cell Signaling Technology); anti-BAX and anti-14-3-3 $\beta$ (monoclonal and polyclonal; Santa Cruz Biotechnology, Santa Cruz, CA); and anti-BCL-X1 (monoclonal; BD Transduction Laboratories, Lexington, KY). Control for protein loading was performed by reprobing membranes with an antibody against $\alpha$-tubulin (Santa Cruz Biotechnology). Membranes were then incubated with appropriate secondary antibodies (1:2000 dilution), followed by chemiluminescence detection (NEN Life Science Products, Boston, MA), and then exposed to Kodak Biomax film (Eastman Kodak, Rochester, NY). Images were collected with a Dage-MTI (Michigan City, IN) 72 camera, and gel bands were analyzed using gel-scanning integrated optical density software (Bioquant, Nashville, TN).

Immunoprecipitation. Animals were killed 0, 4, or $24 \mathrm{hr}$ after administration of diazepam in seizure animals or after $4 \mathrm{hr}$ in nonseizure controls. Pooled brain samples ( $n=4$ animals per group) were dounce homogenized and lysed in buffer containing $1 \% \mathrm{NP}-40$, and the same protease inhibitor cocktail was used for Western blotting. Protein concentration was determined as described previously. Protein samples (0.5 $\mathrm{mg}$ ) were incubated with $2-5 \mu \mathrm{g}$ of the immunoprecipitating antibody overnight at $4^{\circ} \mathrm{C}$ and then incubated with protein $\mathrm{A} / \mathrm{G}$ agarose beads (Santa Cruz Biotechnology) for $2 \mathrm{hr}$ at $4^{\circ} \mathrm{C}$. Additional controls were performed using anti-BID (a gift from Dr. D. Chen, Legacy Research) and anti-Bcl-2 (Santa Cruz Biotechnology). The protein-bead complex was then washed and collected by centrifugation, and samples were boiled in loading buffer and run on $12 \%$ SDS-PAGE gels, probed with the antibody of interest, and processed as described for Western blotting. Positive (50 $\mu \mathrm{g}$ of whole cell lysate) and negative (omitting the immunoprecipitation antibody) controls were included to confirm specificity of reactions.

Immunohistochemistry and DNA fragmentation analysis. Coronal brain sections at the level of bregma $-3.2 \mathrm{~mm}$ (Paxinos and Watson, 1997) from animals killed 4, 24, or $72 \mathrm{hr}$ after seizures or time-matched controls were preblocked in $2 \%$ goat serum and then incubated overnight at $4^{\circ} \mathrm{C}$ with anti-BAD (1:50), anti-BAX (1:200), anti-BCL-X1 (1:100), anti-neuronal-specific nuclear protein (NeuN) (1:500; Chemicon, Temecula, CA), anti-14-3-3 (1:500), or anti-cytochrome IV oxidase (COX IV) (1:200; Molecular Probes, Eugene, OR). Sections were then washed three times in PBS and incubated for $2 \mathrm{hr}$ at room temperature in a 1:500 dilution of goat anti-rabbit or goat anti-mouse AlexaFluor 488 or 568 (Molecular Probes). Sections were then washed and mounted in medium containing 4',6'-diamidino-2-phenylindole (DAPI) (Vector Laboratories, Burlingame, CA) to assess nuclear morphology. Immunolabeling was studied using a Leica (Nussloch, Germany) microscope equipped for epifluorescent illumination under excitation/emission wavelengths of $340 / 425 \mathrm{~nm}$ (blue), 500/550 nm (green), and 580/630 nm (red). Images were collected using an Optronics International (Chelmsford, MA) DEI750 three-chip camera equipped with a BQ 8000 video graphics adaptor frame grabber and analyzed using an image analysis system (Bioquant).

Analysis of cells exhibiting DNA fragmentation was performed using fluorescein- or tetramethylrhodamine-linked terminal deoxynucleotidyl transferase-mediated biotinylated UTP nick end labeling (TUNEL) (Roche Molecular Biochemicals, Indianapolis, IN) to label doublestranded DNA breaks suggestive of apoptosis as described previously (Henshall et al., 2000a,c).

Surviving neurons and TUNEL-labeled cells was averaged from counts on two adjacent brain sections. The hippocampal CA3 subfield was examined in its entirety, whereas cortical counts were the sum of five $40 \times$ fields selected at random within somatosensory cortex.

Immunogold electron microscopy. For ultrastructural localization of BAX in seizure brain samples, animals were transcardially perfused $2 \mathrm{hr}$ after seizure termination with $2 \%$ paraformaldehyde and $0.1 \%$ glutaraldehyde in 0.1 M PBS. Brains were extracted, microdissected, postfixed, and embedded in plastic. Ultrathin sections from the injured ipsilateral CA3 subfield were prepared on a microtome, and then samples were stained overnight at $4^{\circ} \mathrm{C}$ with anti-BAX, followed by $2 \mathrm{hr}$ at room temperature with goat anti-rabbit immunogold conjugate (particle size, 15 nm; Ted Pella, Redding, CA). Sections were then examined using a transmission EM (EM 10CA; Zeiss, Oberkochen, Germany).

Data analysis. Data are presented as mean \pm SEM. Data were analyzed using one-way ANOVA with appropriate post hoc tests (StatView software; SAS Institute, Cary, NC). Significance was accepted at $p<0.05$.

\section{RESULTS}

\section{Seizures induce CA3 pyramidal neuron death with features of apoptosis}

Twenty-four hours after seizures evoked by intraamygdala KA, $\sim 70 \%$ of ipsilateral CA3 pyramidal neurons had degenerated (Fig. $1 B c)$, and many $(107 \pm 7)$ were positively labeled for TUNEL (Fig. $1 B d)(n=4)$. Small numbers of these TUNELlabeled cells exhibited features of apoptosis (Fig. $1 \mathrm{Bd}$, inset). In contrast, the ipsilateral cortex exhibited only small numbers of TUNEL-labeled cells $(16 \pm 3)$ after seizures, and TUNELpositive cells were not detected within contralateral brain regions (data not shown).

\section{Expression of BAD pathway components after seizures}

To confirm the expression of the key signaling components in this pathway and to establish that alterations in protein-protein interactions are not a consequence of underlying changes in protein levels, we performed Western blotting. All components in the BAD signaling pathway, including 14-3-3, BAD, BCL-X1, and BAX, were constitutively expressed in brain ( $n=4$ per group). Seizures did not affect levels of each of the key components of the pathway to be examined within ipsilateral hippocampus (Fig. 2) or cortex (data not shown). 
A

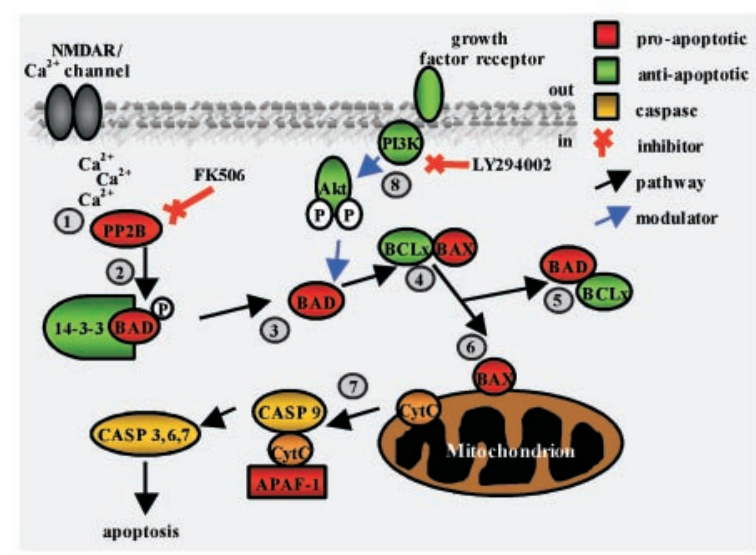

B

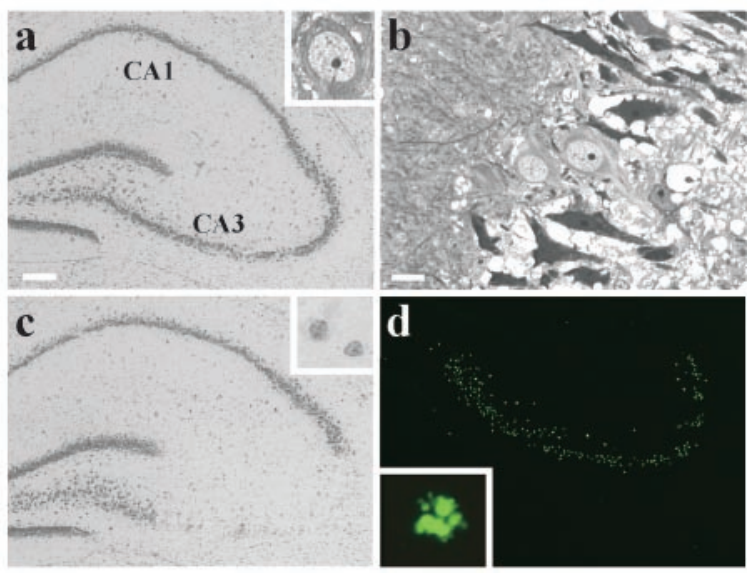

Figure 1. BAD pathway and seizure-induced neuronal injury characteristics. $A$, Schematic depicting BAD cell death pathway, its binding interaction changes, and putative pharmacological manipulation. The pathway is thought to be engaged after the following: (1) calcium $\left(\mathrm{Ca}^{2+}\right)$ entry, which activates the phosphatase calcineurin, which then (2) dephosphoryates BAD. Released BAD (3) then interacts with (4) the BCL-X:BAX complex and displaces BAX, resulting in (5) a BAD:BCL-X dimer. (6) BAX subsequently translocates to the mitochondrion, in which it triggers (7) cytochrome $c$ release and formation of the apoptosome, which results in apoptosis. In turn, sequestration of BAD to 14-3-3 may be restored by phosphorylation downstream of (8) Akt. NMDAR, NMDA receptor; $P$, phosphate group; $P P 2 B$, protein phosphatase 2B/calcineurin; $C A S P$, caspase; Akt, protein kinase B; PI3K, PI3-kinase; Cyt $c$, cytochrome $c$; $A P A F-1$, apoptotic protease-activating factor 1 . $B$, Histopathology of seizure model. $a$, Photomicrograph showing control ipsilateral hippocampus of the rat. Inset, High-power magnification of a single CA3 pyramidal neuron. $b$, Toluidine blue-stained CA3 field revealing early (4 hr) degenerative changes in some CA3 neurons. $c$, Photomicrograph $24 \mathrm{hr}$ after seizures revealing marked loss of CA3 pyramidal neurons. Inset, Shrunken, condensed nuclei of two CA 3 cells. $d$, Low-power field showing extensive TUNEL labeling (DNA fragmentation) throughout the degenerating CA3 subfield. Inset, View of a CA3 TUNEL-labeled cell exhibiting nuclear features of apoptosis. Scale bar: $a, c, d, 200 \mu \mathrm{m} ; b, 15 \mu \mathrm{m}$.

\section{Dissociation of BAD from 14-3-3 after seizures}

We first examined the interaction of BAD with the molecular chaperone 14-3-3 using immunoprecipitation. Preliminary studies confirmed that the BAD antibody effectively precipitated BAD from rat brain hippocampus (Fig. $3 A$ ). Next, we immunoprecipitated $\mathrm{BAD}$ and then performed Western blotting to detect the

\section{hippocampus}

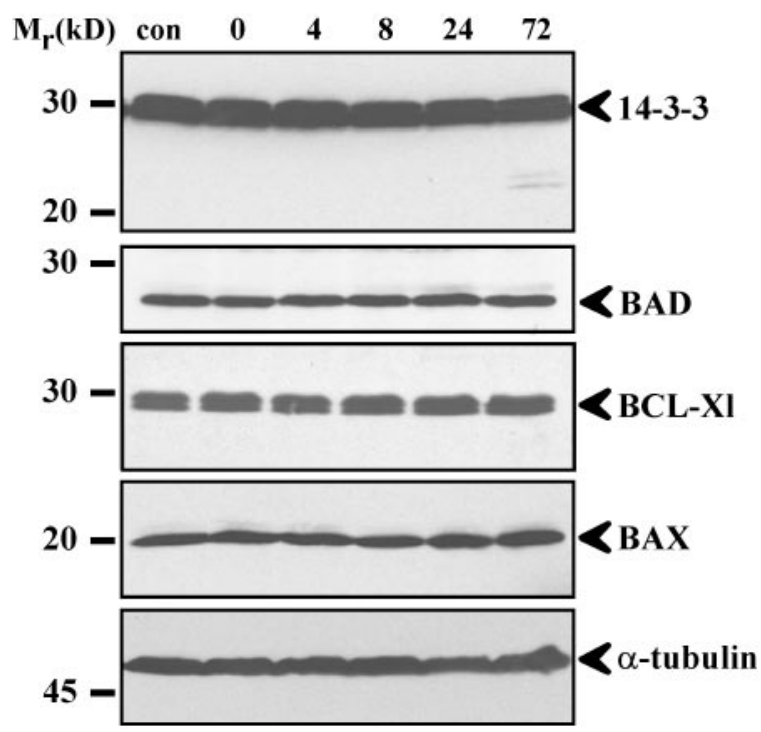

Figure 2. Expression of BAD cell death pathway components after seizures. Representative Western blots $(n=2$ per lane) showing expression of key components of BAD pathway within ipsilateral hippocampus. Seizures had no obvious effect on expression levels of any of the BAD pathway components. Protein-loading controls are shown for $\alpha$-tubulin. Molecular weight markers are depicted to the left. Immunoblots are representative of at least two independent experiments.

presence of 14-3-3. In control animals, we detected a robust constitutive interaction between BAD and 14-3-3 in ipsilateral hippocampus (Fig. 3B). In contrast, binding of 14-3-3 to BAD decreased after seizures, and semiquantification of protein levels determined that this interaction was significantly reduced $24 \mathrm{hr}$ after seizures (Fig. 3C). Binding of BAD to 14-3-3 within ipsilateral cortex after seizures did not change (data not shown). Studies have suggested that binding interactions of Bcl-2 family proteins may be erroneous because of inclusion of detergents in lysis buffer (Hsu and Youle, 1997). Therefore, we repeated this immunoprecipitation reaction with or without detergent and determined that NP-40 does not increase the binding of BAD to 14-3-3 (data not shown). As a final control experiment, we examined the interaction of BID, a proapoptotic BAD homolog, with 14-3-3. BID was immunoprecipitated from rat brain and then immunoblotted with the antibody against 14-3-3. In contrast to BAD, we did not detect any 14-3-3 bound to BID in control brain (data not shown).

Immunohistochemical colocalization of BAD with 14-3-3 and the nuclear translocation of 14-3-3 after seizures

To support our immunoprecipitation findings, we examined BAD and 14-3-3 expression in brain sections from control animals or 4 and $24 \mathrm{hr}$ after seizure termination ( $n=4$ per group) (Fig. 4 ). Expression of BAD in control brain was detected in many neurons throughout the brain, including the hippocampal CA subfields, cortex, thalamus, and also abundantly within the epithelial cells of the choroid plexus. Staining was primarily cytosolic and appeared punctate in some cells. Expression of 14-3-3 was also ubiquitous throughout cortex and hippocampus in control brain. Examination of BAD and 14-3-3 expression within the same cells determined that there was constitutive overlap between these proteins under control conditions, although it was not complete (Fig. 4A-C).

Twenty-four hours after seizures, the distribution of 14-3-3 
A
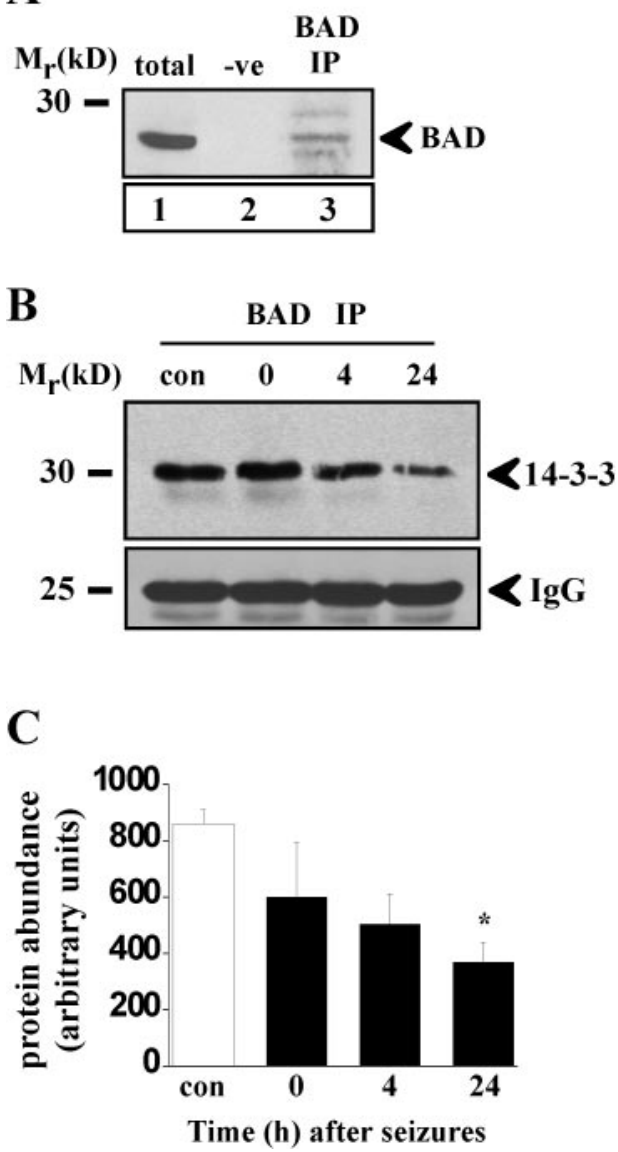

Figure 3. Dissociation of BAD from 14-3-3 after seizures. $A$, Representative Western blot ( $n=2$ per lane) to demonstrate effectiveness of BAD antibody to precipitate BAD. Lane 1, BAD in rat brain (total); lane 2, negative control $(-v e)$ in which the immunoprecipitation antibody was omitted; lane 3, confirmation that the $\mathrm{BAD}$ antibody effectively precipitated BAD from rat brain $(B A D I P)$. B, Representative Western blot showing decline of 14-3-3 binding to BAD after seizures ( $n=4$ per lane) within ipsilateral hippocampus. IgG bands are shown to confirm equality of antibody loading. $C$, Semiquantification of BAD interaction with 14-3-3 within ipsilateral hippocampus confirming decreased binding of BAD to 14-3-3 after seizures. Data are from two independent experiments. ${ }^{*} p<$ 0.05 compared with control.

changed dramatically within the selectively vulnerable neurons of the ipsilateral CA3, becoming almost exclusively nuclear, suggesting translocation of 14-3-3 after seizures (Fig. 4D). In contrast, BAD staining within such CA3 neurons was typically very low or appeared as cytoplasmic puncta (Fig. 4E,F). To extend these observations, we examined DNA fragmentation staining within those neurons in which 14-3-3 appeared nuclear. We found that nuclear 14-3-3 was present exclusively in TUNEL-positive CA3 cells (Fig. 4G-I).

\section{Seizures induce binding of BAD to BCL-XI}

To examine whether BAD interacts with BCL-X1 after its release from 14-3-3, we used the BAD antibody to immunoprecipitate $\mathrm{BAD}$ and probed for $\mathrm{BCL}-\mathrm{Xl}$. We detected $\mathrm{BCL}-\mathrm{Xl}$ at very low levels in control brain BAD immunoprecipitates (Fig. $5 A$ ). Semiquantification of protein levels (Fig. $5 B$ ) determined that, $4 \mathrm{hr}$ after seizures, there was a significant increase in levels of BCL-X1 binding to BAD compared with control and levels at $0 \mathrm{hr}$. Levels of BCL-Xl binding to BAD were also elevated at $24 \mathrm{hr}$, although
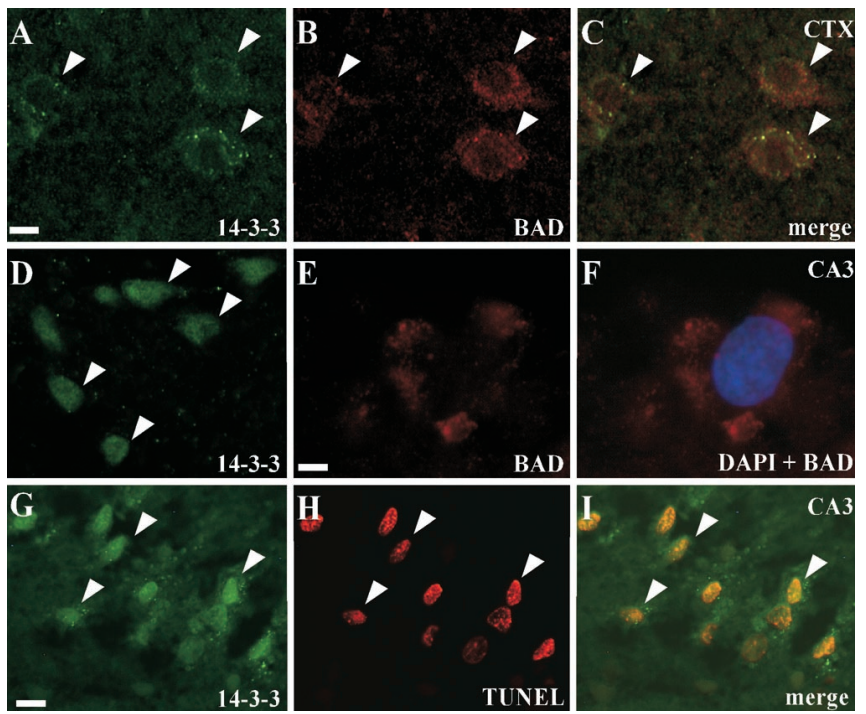

Figure 4. Immunohistochemical colocalization of BAD with 14-3-3 and their dissociation after seizures. $A$, Control expression of 14-3-3 was detected throughout cortex $(C T X)$ and colocalized substantially with BAD $(B)$ as evidenced by image overlay $(C)$. Twenty-four hours after seizures, 14-3-3 expression $(D)$ became almost exclusively nuclear within CA3 pyramidal neurons. In contrast, examination of a single BAD-labeled CA3 cell $(E, F)$ revealed punctate staining that remained within the cytoplasm. Counterstaining of 14-3-3-labeled sections $(G)$ with the DNA fragmentation marker TUNEL $(H)$ revealed that nuclear 14-3-3 was exclusively within dying cells. Arrowheads indicate representative immunopositive cells. Scale bars: $A-C, D, 15 \mu \mathrm{m} ; E, F, 4 \mu \mathrm{m} ; G-I, 18 \mu \mathrm{m}$.

this did not reach statistical significance. No change in BAD: BCL-X1 binding was detected in ipsilateral cortex after seizures (data not shown). To confirm the specificity of this interaction, we reprobed membranes to detect $\mathrm{Bcl}-2$. We did not detect $\mathrm{Bcl}-2$ bound to precipitated BAD in control or seizure brain at any time (data not shown).

We next used immunohistochemistry to examine this interaction within affected CA3 cells ( $n=4$ per group). We detected constitutive expression of BCL-Xl in rat brain cortex and hippocampus in control animals. Examination of brain sections from animals $4 \mathrm{hr}$ after seizures revealed increased colocalization of BCL-X1 with BAD within many CA3 neurons (data not shown), and, in sections $24 \mathrm{hr}$ after seizures, BCL-Xl strongly colocalized with BAD in some but not all injured-dying CA3 neurons (Fig. 5C).

\section{Mitochondrial BAX accumulation after seizures}

BAX has been shown to reside in an inactive complex with $\mathrm{BCL}-\mathrm{Xl}$ under control conditions, and so the binding of BAD to $\mathrm{BCL}-\mathrm{Xl}$ is thought to trigger $\mathrm{BAX}$ displacement. Using anti$\mathrm{BAX}$ as the immunoprecipitating antibody, we detected robust constitutive binding of BAX to BCL-Xl in control brain $(n=4$ per group), but we did not detect a significant change in BAX:BCL-X1 binding after seizures in either ipsilateral hippocampus or cortex (data not shown).

Because BAX release-activation could not be detected by this method, we examined BAX localization within CA3 neurons by immunohistochemistry ( $n=4$ per group). BAX immunostaining in control brain was very low but nevertheless ubiquitous in neurons and glia. BAX appeared most often as a fine granular pattern similar to that of control BCL-X1 that was primarily restricted to the nucleus. BAX immunostaining did not overlap 
A

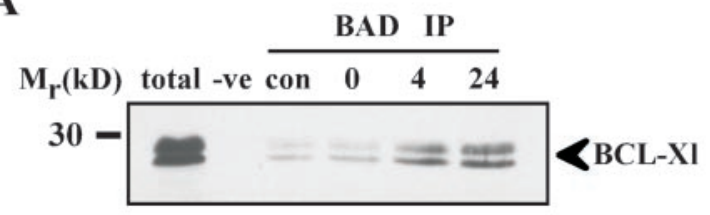

B

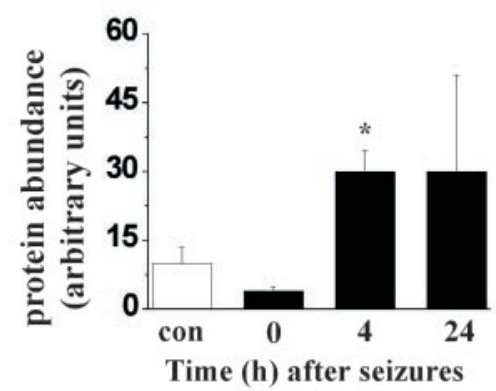

C
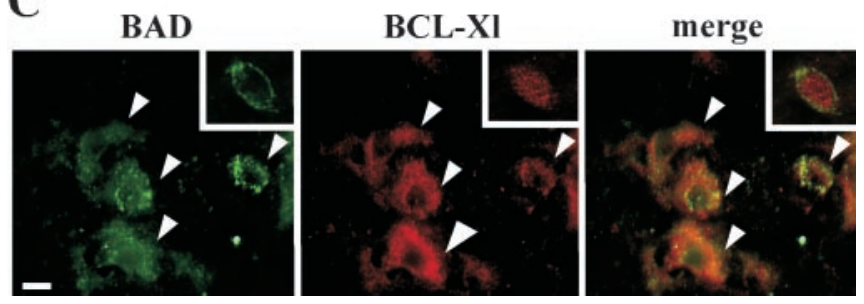

Figure 5. Increased binding of BAD to BCL-X1 after seizures. $A$, Representative Western blot $(n=4$ per lane) showing increased binding of BCL-X1 to BAD immunoprecipitates $(B A D I P) 4 \mathrm{hr}$ after seizures within ipsilateral hippocampus compared with control (con). Whole brain lysate (total) and omission of the immunoprecipitated antibody $(-v e)$ are shown to confirm reaction specificity. $B$, Semiquantification of increased BAD interaction with BCL-X1 after seizures from two independent experiments. ${ }^{*} p<0.05$ compared with control. $C$, Immunohistochemical colocalization of BAD (green) and BCL-X1 (red) $24 \mathrm{hr}$ after seizures within $\mathrm{CA} 3$ as revealed by yellow-orange coloring in merge panel. Insets show corresponding control expression of each protein. Arrowheads indicate representative immunopositive cells. Scale bar, $15 \mu \mathrm{m}$.

with the mitochondrial marker COX IV in control CA3 cells (Fig. $6 \mathrm{Aa}$ ). Four and $24 \mathrm{hr}$ after seizures, punctate BAX immunostaining was detected within the cytoplasm of affected CA3 neurons (Fig. 6Ab-Ad), and BAX staining colocalized to some degree with the mitochondrial marker COX IV in $80 \%$ of neurons examined (from 50 neurons studied within $n=3$ brains).

To further establish the mitochondrial accumulation of BAX at the ultrastructural level, we examined BAX distribution by immunogold labeling using electron microscopy at the time shown previously to coincide with cytochrome $c$ release in this model (Henshall et al., 2000a). Examination of hippocampal fields after seizures revealed prominent BAX particle accumulation surrounding mitochondrial membranes (Fig. 6B). BAX particles could also be detected on the inner nuclear membrane, but BAX was not present on other organelle membranes, such as the endoplasmic reticulum (data not shown).

\section{Effects of FK506 on seizure-induced brain injury}

FK506 is a calcineurin inhibitor that has been shown to be neuroprotective after neuronal injury in vivo (Butcher et al., 1997; Moriwaki et al., 1998), likely through blocking dephosphorylation of BAD (Springer et al., 2000). To better address the contribution
A
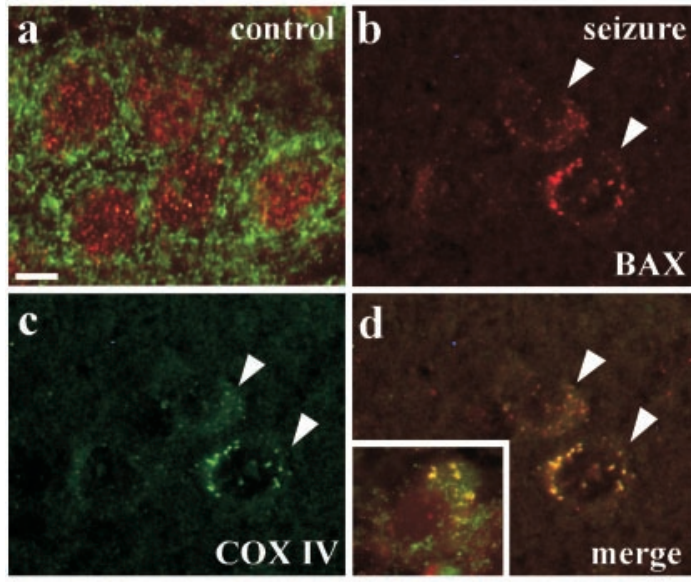

B

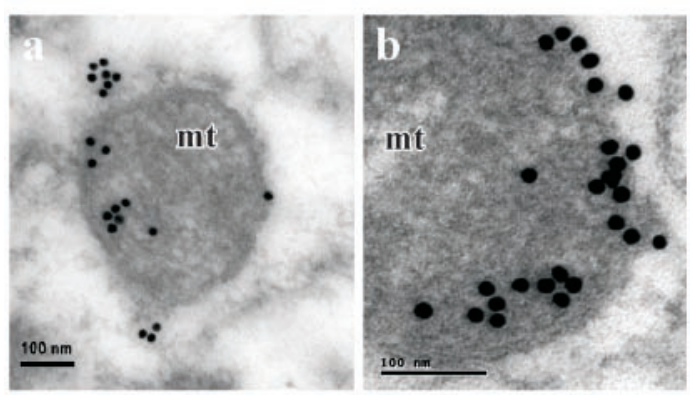

Figure 6. BAX accumulation at mitochondria after seizures. $A$, Immunohistochemical detection of $\operatorname{BAX}(a ; r e d)$ and the mitochondrial marker COXIV (green) within control brain CA3 subfield in which no colocalization is detected. Twenty-four hours after seizures, BAX $(b)$ appeared as a punctate stain within affected CA3 cells and exhibited marked colocalization with COXIV $(c)$ as revealed by yellow-gold puncta in $d$, the merge panel. Inset in $d$ shows colocalization at $4 \mathrm{hr}$ in which overlap is detectable but not as extensive. Arrowheads indicate representative immunopositive cells. Scale bar, $15 \mu \mathrm{m}$. $B$, Representative electron micrographs showing ( $a$, low-power field; $b$, high-power field) BAX accumulation at mitochondrial ( $m t$ ) membranes $2 \mathrm{hr}$ after seizures as labeled by 15 $\mathrm{nm}$ immunogold particles. Note clustering of BAX at outer surface of mitochondria.

of BAD to seizure-induced neuronal death, we examined the effect of FK506 in our model. The duration of injury-producing seizures in animals that received FK506 (239 $\pm 96 \mathrm{sec}$ ) was not significantly different from that in vehicle-injected seizure animals $(200 \pm 82 \mathrm{sec})$, and F K506 had no significant effect on the onset or offset of seizures in this model. Seizures induced marked TUNEL labeling and death of ipsilateral CA3 pyramidal neurons when examined $72 \mathrm{hr}$ after seizures (Fig. $7 A$ ). The phenotype of CA3 region TUNEL-positive cells was $>99 \%$ neuronal as determined by counterstaining sections with NeuN (from 691 cells counted from five brains). FK506 significantly reduced numbers of TUNEL-positive cells within CA3 by $49 \%$ (Fig. $7 A$ ) and improved neuronal survival by $26 \%$ after seizures (Fig. $7 B$ ).

\section{Activation of Akt after seizures}

Phosphorylated Akt may protect neurons from cell death by inducing BAD phosphorylation and sequestration to 14-3-3 (Datta et al., 1997). Furthermore, Akt can be activated after 
A

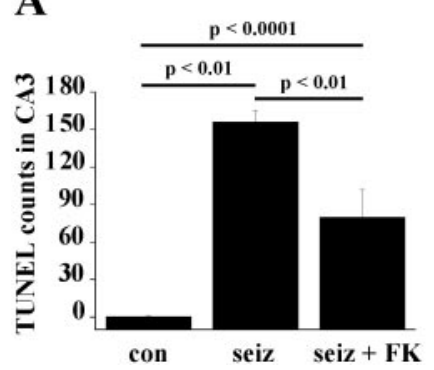

B

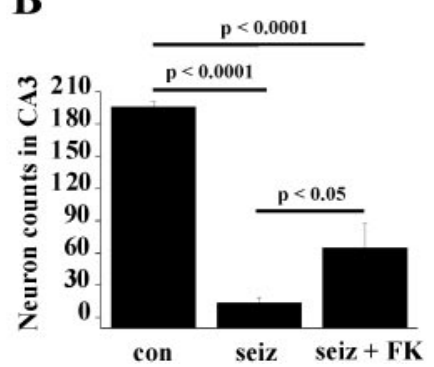

Figure 7. Effects of FK506 on cell survival. A, Graph showing quantification of hippocampal CA3 TUNEL labeling in control (con) and seizure (seiz) animals injected with vehicle or FK506 ( $n=6$ per group). Seizures induced significant TUNEL labeling within CA 3 cells $72 \mathrm{hr}$ after diazepam compared with nonseizure controls. FK506 (seiz $+F K)$ significantly reduced numbers of TUNEL-positive cells compared with vehicle-treated seizure animals. $B$, Seizures induced a significant reduction in numbers of CA3 neurons after seizures, and this was partly blocked by FK506.

survival factor (e.g., brain-derived neurotrophic factor) release, a well documented consequence of seizures (Elmer et al., 1998; Katoh-Semba et al., 1999; Reibel et al., 2001). Because seizureinduced cell death and activation of BAD-BAX was most extensive within the hippocampus, whereas cortex was primarily spared, we sought to determine whether differences in Akt phosphorylation existed between these brain regions. We detected strong constitutive expression of Akt by Western blotting within both hippocampus and cortex under control conditions $(n=4$ per group), and levels were unaffected by seizures in either brain region (Fig. 8A,B). Within ipsilateral cortex, Western blotting determined that Akt was rapidly phosphorylated at $\mathrm{Thr}^{308}$ and $\mathrm{Ser}^{473}$ residues after seizures (Fig. $8 B$ ). In contrast, no changes in Akt phosphorylation were detected within ipsilateral hippocampus above background (control) levels at any time after seizures (Fig. 8A).

Using the phosphoAkt ${ }^{473}$ antibody, we performed immunohistochemical analysis of Akt activation on brain sections from control or seizure animals ( $n=4$ per group). Under control conditions, phosphoAkt ${ }^{473}$ staining was very low throughout cortex (Fig. 8Ca) and hippocampus (data not shown). Twenty-four hours after seizure termination, increased levels of phosphoAkt ${ }^{473}$ were detected throughout ipsilateral cortex (Fig. $8 C b$ ), whereas levels within the injured hippocampal CA3 remained similar to control (data not shown).

If Akt contributes to the protection of the cortex from seizures, we reasoned that blocking Akt activation should exacerbate cortical injury. LY294002 is a PI3 kinase inhibitor that blocks Akt activation in vivo (Noshita et al., 2001); therefore, we examined the effect of intracerebroventricular administration of LY294002. The duration of injury-producing seizures in animals infused with $100 \mathrm{nmol}$ of LY294002 (114 $\pm 50 \mathrm{sec})$ was not significantly different from that in vehicle-injected seizure controls $(98 \pm 28$ sec). LY294002 significantly increased numbers of cortical TUNEL-positive cells compared with vehicle-treated seizure controls (Fig. $8 D b$ ) but had no effect on cell death within injured hippocampus (Fig. 8Da).

\section{DISCUSSION}

In the present study, we determined that seizures triggered the dissociation of the death agonist BAD from 14-3-3 within the damaged hippocampus. Subsequently, 14-3-3 translocated to the nucleus, whereas BAD went on to interact with BCL-X1, and
A hippocampus B cortex
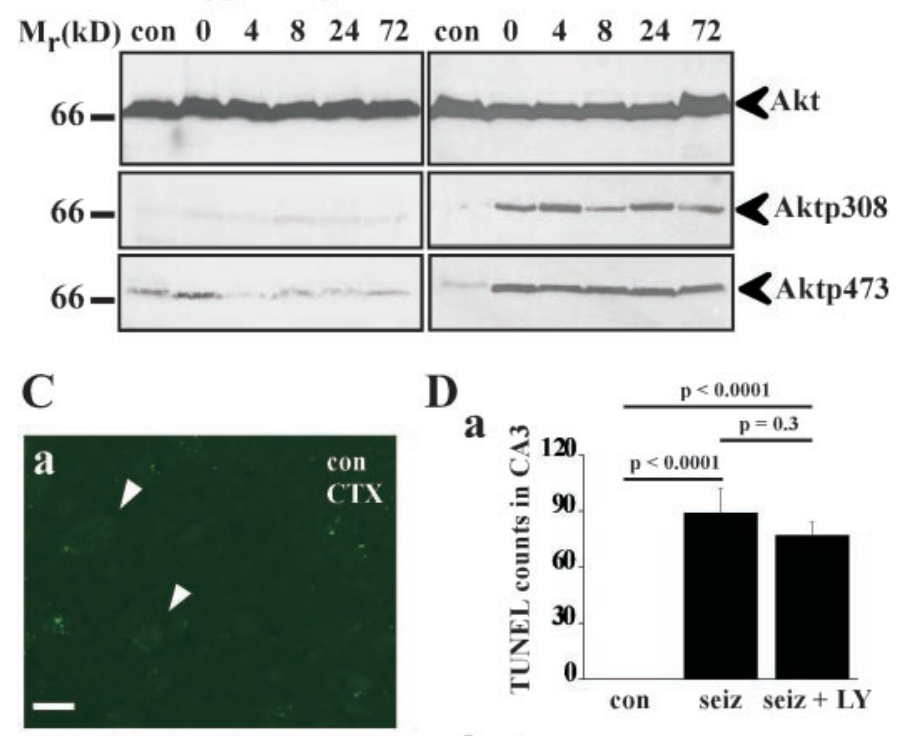

D
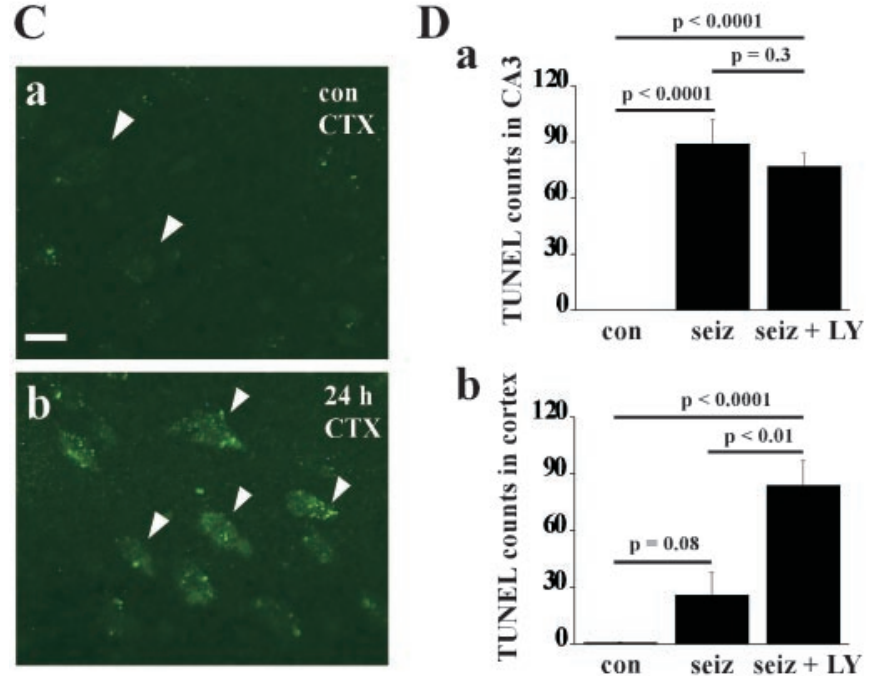

b

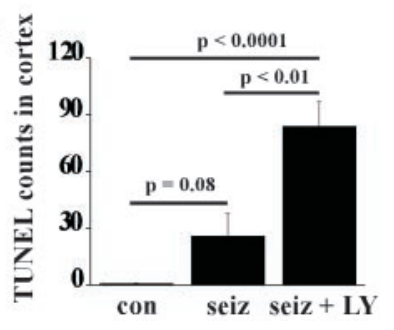

Figure 8. Akt activation in surviving brain regions after seizures. $A$, Representative Western blots $(n=2$ per lane) showing Akt expression and phosphoAkt $\mathrm{Thr}^{308}$ and $\mathrm{Ser}^{473}$ levels in ipsilateral hippocampus after seizures. No activation of Akt was detected after seizures. B, Representative Western blots ( $n=2$ per lane) showing Akt expression and rapid phosphorylation at both consensus sites within cortex after seizures. $C$, Immunohistochemical detection of phosphoAkt $\mathrm{Ser}^{473}$ in rat brain revealed low to undetectable levels of phosphoAkt ${ }^{473}$ within control cortex (a). $b$, Twenty-four hours after seizures, phosphoAkt ${ }^{473}$ was upregulated within many cells within cortex $(C T X)$. Arrowheads indicate representative immunopositive cells. Scale bar (in $a$ ): $20 \mu \mathrm{m}$. $D$, Quantification of TUNEL counts $72 \mathrm{hr}$ after seizures in control animals (con) or seizure rats inf used with vehicle (seiz) or the PI3-kinase inhibitor LY294002 (seiz $+L Y)(n=5$ per group). $a$, Seizures induced significant hippocampal CA3 TUNEL labeling compared with nonseizure controls in both groups, but there was no significant difference between vehicle and LY294002 groups. In contrast, LY294002 (b) significantly increased numbers of TUNEL counts within cortex compared with both nonseizure controls and vehicle-treated seizure animals.

BAX accumulated at mitochondrial membranes. In contrast, the primarily undamaged cortex exhibited prosurvival responses, such as Akt phosphorylation. The functional significance of the BAD pathway was supported by the complementary findings that an inhibitor of the BAD pathway was neuroprotective, whereas blocking the (protective) effects of Akt exacerbated damage. These data suggest that BAD may be an initiation or reinforcement step in the process of cell death after seizures.

To date, there has been no functional assessment of the Bcl-2 family protein response to seizures, with only descriptive reports on transcription and expression levels of some of the Bcl-2 family proteins in experimental models and human temporal lobe epilepsy (Gillardon et al., 1995; Lopez et al., 1999; Tuunanen et al., 1999; Henshall et al., 2000b). Presently, we examined the functional responses of the BAD pathway as a possible trigger for 
neuronal death after seizures. Our coimmunoprecipitation studies confirmed that both BAD:14-3-3 and BCL-X1:BAX complexes are constitutively present in rat brain and demonstrated dissociation of BAD from 14-3-3 and binding to BCL-Xl after seizures in the hippocampus. Because changes in expression levels were not detected for these proteins, this cannot be explained by seizures inducing upregulation-downregulation of these proteins, and, therefore, these changes represent the first demonstration of seizure-induced activation of the BAD pathway. These interaction changes were also confirmed by immunohistochemistry in which both the resting interactions of BAD, 14-3-3, and BCL-X1, as well as their seizure-induced conformations, were observed. Corresponding reductions in levels of the BCL-X1:BAX complex after seizures were not apparent in our studies perhaps because of the high constitutive interaction levels along with relatively small quantities of BAX release that could place such observations beyond the sensitivity of this assay technique. BAX reaction to seizures was inferred nonetheless by immunohistochemistry and immunogold electron microscopic detection of BAX accumulation around mitochondria. Therefore, these data suggest that BAD may be an instigator of BAXfacilitated cytochrome $c$ release after seizures. However, other neuronally expressed proapoptotic Bcl-2 proteins such as Bcl-2homologous antagonist/killer may also be important in triggering cytochrome $c$ release (Sun et al., 2001; Wei et al., 2001). Therefore, additional studies are required to determine the relative importance of BAX downstream of BAD activation in relation to seizure-induced mitochondrial dysfunction.

Immunohistochemistry revealed the nuclear translocation of 14-3-3 proteins in dying (TUNEL positive) CA3 neurons after seizures. To our knowledge, this is the first demonstration of this event after seizures. Although the 14-3-3 antibody used preferentially recognizes the $\beta$-isoform, the likely cross-reactivity to other isoforms (Fig. 2, doublet band) precludes additional insight into which 14-3-3 isoform is critical to these interaction changes with BAD. 14-3-3 isoforms are expressed ubiquitously in rat brain, although their subcellular localizations exhibit differences (Martin et al., 1994), and all isoforms interact with BAD (Subramanian et al., 2001). A functional consequence of the nuclear localization of 14-3-3 after seizures is not known but implied by the delay between the immunoprecipitation interaction changes of BAD with 14-3-3 and BCL-X1 (detected $4 \mathrm{hr}$ after seizure) and appearance of 14-3-3 in nucleus (at $24 \mathrm{hr}$ but not $4 \mathrm{hr}$ ). The 14-3-3 family proteins interact with a number of proteins that could drive this nuclear targeting, including p53, which induces apoptosis in response to stimuli, such as DNA damage (Waterman et al., 1998). Both p53 nuclear activation and DNA damage are well documented after seizures (Sakhi et al., 1996; Liu et al., 1999). However, 14-3-3 isoforms interact with a number of nuclear receptors (Zilliacus et al., 2001) and may localize to the nucleus under other conditions (Peng et al., 1997; Todd et al., 1998). Thus, additional studies are required to elucidate the significance of 14-3-3 nuclear translocation after brain injury.

Contrasting events in hippocampus, the cortex exhibited minimal TUNEL labeling after seizures. Using immunoblotting and immunohistochemistry, we determined that this region is not associated with activation of the BAD pathway but rather exhibits predominantly survival responses, including Akt phosphorylation. Akt phosphorylation is a survival-promoting event reported previously after ischemic brain injury (Ouyang et al., 1999; Noshita et al., 2001; Yano et al., 2001), likely downstream of growth factor receptors (Franke et al., 1997), which may be activated by seizures (Elmer et al., 1998). Activated Akt has a number of substrates, including the BAD Ser ${ }^{136}$ site of which phosphorylation would drive BAD sequestration to 14-3-3 (Masters et al., 2001). The antibodies used in the present studies detect those consensus sites of the $\mathrm{Akt} 1 / \mathrm{PKB} \alpha$ isoform that is expressed in brain and phosphorylated by 3-phosphoinositide-dependent kinase-1, although other Akt isoforms have similar consensus sequences (Kandel and Hay, 1999). Both $\mathrm{Thr}^{308}$ and $\mathrm{Ser}^{473}$ consensus sites on cortically expressed Akt exhibited increased phosphorylation after seizures in our studies, whereas the hippocampus exhibited no change in Akt phosphorylation. The functional significance of Akt activation was supported by the demonstration that the putative PI3 kinase inhibitor LY294002 exacerbated cortical cell death. Therefore, these data support a protective effect of Akt activation in limiting seizure-induced cell death, perhaps through inhibition of BAD. However, activated Akt has a number of other substrates associated with cell survival (Cross et al., 2000), and the resistance of the cortex to seizureinduced cell death may also reside with differences in neuroanatomy, neurotransmitter receptor expression, and cell death modulatory pathways in addition to Akt. Nevertheless, activation of the Akt survival pathway to intervene in proapoptotic pathway engagement after seizures may yield therapeutic strategies for the treatment of seizure-induced brain injury.

Although the BAD pathway was activated after seizures, the time lag relative to BID activation, which may be detected even earlier (0-2 hr after seizure) (Henshall et al., 2001b), suggests that BAD may either simply reinforce events initiated by BID or even be redundant. Supporting a substantive effect of BAD, we found the calcineurin inhibitor F K506 reduced the appearance of TUNEL-DNA fragmentation and neuronal death after seizures. FK506 has been shown previously to block BAD dephosphorylation and cell death in other neuronal injury models (Springer et al., 2000), and FK506 has been shown to protect the brain from systemic kainic acid neurotoxicity (Moriwaki et al., 1998). Because FK506 may have protective effects in addition to those mediated by interrupting the BAD pathway (Snyder et al., 1998), we cannot exclude other explanations for the present data; however, these results suggest that BAD does contribute to cell death after seizures.

The present data and previous work establish that seizures activate multiple cell death pathways involving Bcl-2 and caspase family proteins in brain regions destined to die, whereas survivalpromoting responses predominate in cortical populations that survive. Proapoptotic BAD and the counteractive effects of Akt may underlie in part the cell death outcome after seizures, providing a more complete understanding of the mechanisms by which seizures damage brain and highlighting novel targets for treatment of brain injury associated with seizure disorders.

\section{REFERENCES}

Adams JM, Cory S (1998) The Bcl-2 protein family: arbiters of cell survival. Science 281:1322-1326.

Bengzon J, Kokaia Z, Elmer E, Nanobashvili A, Kokaia M, Lindvall O (1997) Apoptosis and proliferation of dentate gyrus neurons after single and intermittent limbic seizures. Proc Natl Acad Sci USA 94:10432-10437.

Bratton SB, Walker G, Srinivasula SM, Sun XM, Butterworth M, Alnemri ES, Cohen GM (2001) Recruitment, activation and retention of caspases- 9 and -3 by Apaf-1 apoptosome and associated XIAP complexes. EMBO J 20:998-1009.

Burgering BM, Coffer PJ (1995) Protein kinase B (c-Akt) in phosphatidylinositol-3-OH kinase signal transduction. Nature 376:599-602.

Butcher SP, Henshall DC, Teramura Y, Iwasaki K, Sharkey J (1997) Neuroprotective actions of FK506 in experimental stroke: in vivo evidence against an antiexcitotoxic mechanism. J Neurosci 17:6939-6946. 
Cross TG, Scheel-Toellner D, Henriquez NV, Deacon E, Salmon M, Lord JM (2000) Serine/threonine protein kinases and apoptosis. Exp Cell Res 256:34-41.

Datta SR, Dudek H, Tao X, Masters S, Fu H, Gotoh Y, Greenberg ME (1997) Akt phosphorylation of BAD couples survival signals to the cell-intrinsic death machinery. Cell 91:231-241.

Datta SR, Katsov A, Hu L, Petros A, Fesik SW, Yaffe MB, Greenberg ME (2000) 14-3-3 proteins and survival kinases cooperate to inactivate BAD by BH3 domain phosphorylation. Mol Cell 6:41-51.

Dudek H, Datta SR, Franke TF, Birnbaum MJ, Yao R, Cooper GM, Segal RA, Kaplan DR, Greenberg ME (1997) Regulation of neuronal survival by the serine-threonine protein kinase Akt. Science 275:661-665

Elmer E, Kokaia Z, Kokaia M, Carnahan J, Nawa H, Lindvall O (1998) Dynamic changes of brain-derived neurotrophic factor protein levels in the rat forebrain after single and recurring kindling-induced seizures. Neuroscience 83:351-362.

Franke TF, Kaplan DR, Cantley LC (1997) PI3K: downstream AKTion blocks apoptosis. Cell 88:435-437.

Fujikawa DG, Shinmei SS, Cai B (2000a) Kainic acid-induced seizures produce necrotic, not apoptotic, neurons with internucleosomal DNA cleavage: implications for programmed cell death mechanisms. Neuroscience 98:41-53.

Fujikawa DG, Shinmei SS, Cai B (2000b) Seizure-induced neuronal necrosis: implications for programmed cell death mechanisms. Epilepsia 41:S9-S13.

Gillardon F, Wickert H, Zimmermann M (1995) Up-regulation of bax and down-regulation of bcl-2 is associated with kainate-induced apoptosis in mouse brain. Neurosci Lett 192:85-88.

Henshall DC, Chen J, Simon RP (2000a) Involvement of caspase-3-like protease in the mechanism of cell death following focally evoked limbic seizures. J Neurochem 74:1215-1223.

Henshall DC, Clark RS, Adelson PD, Chen M, Watkins SC, Simon RP (2000b) Alterations in bcl-2 and caspase gene family protein expression in human temporal lobe epilepsy. Neurology 55:250-257.

Henshall DC, Sinclair J, Simon RP (2000c) Spatio-temporal profile of DNA fragmentation and its relationship to patterns of epileptiform activity following focally evoked limbic seizures. Brain Res 858:290-302.

Henshall DC, Bonislawski DP, Skradski SL, Araki T, Lan J-Q, Schindler CK, Meller R, Simon RP (2001a) Formation of the Apaf-1/cytochrome c complex precedes activation of caspase-9 during seizureinduced neuronal death. Cell Death Differ 8:1169-1181.

Henshall DC, Bonislawski DP, Skradski SL, Meller R, Lan J-Q, Simon RP (2001b) Cleavage of Bid may amplify caspase-8-induced neuronal death following focally evoked limbic seizures. Neurobiol Dis 8:568-580.

Hsu YT, Youle RJ (1997) Nonionic detergents induce dimerization among members of the Bcl-2 family. J Biol Chem 272:13829-13834.

Kandel ES, Hay N (1999) The regulation and activities of the multifunctional serine/threonine kinase Akt/PKB. Exp Cell Res 253:210-229.

Katoh-Semba R, Takeuchi IK, Inaguma Y, Ito H, Kato K (1999) Brainderived neurotrophic factor, nerve growth and neurotrophin-3 selected regions of the rat brain following kainic acid-induced seizure activity. Neurosci Res 35:19-29.

Korsmeyer SJ, Wei MC, Saito M, Weiler S, Oh KJ, Schlesinger PH (2000) Pro-apoptotic cascade activates BID, which oligomerizes BAK or BAX into pores that result in the release of cytochrome c. Cell Death Differ 7:1166-1173.

Kroemer G, Reed JC (2000) Mitochondrial control of cell death. Nat Med 6:513-519.

Li P, Nijhawan D, Budihardjo I, Srinivasula SM, Ahmad M, Alnemri ES, Wang X (1997) Cytochrome $c$ and dATP-dependent formation of Apaf-1/caspase-9 complex initiates an apoptotic protease cascade. Cell 91:479-489.

Liu W, Rong Y, Baudry M, Schreiber SS (1999) Status epilepticus induces p53 sequence-specific DNA binding in mature rat brain. Brain Res Mol Brain Res 63:248-253.

Lizcano JM, Morrice N, Cohen P (2000) Regulation of BAD by cAMPdependent protein kinase is mediated via phosphorylation of a novel site, Ser155. Biochem J 349:547-557.

Lopez E, Pozas E, Rivera R, Ferrer I (1999) Bcl-2, Bax and Bcl-x expression following kainic acid administration at convulsant doses in the rat. Neuroscience 91:1461-1470.

Martin H, Rostas J, Patel Y, Aitken A (1994) Subcellular localisation of 14-3-3 isoforms in rat brain using specific antibodies. J Neurochem 63:2259-2265.

Masters SC, Yang H, Datta SR, Greenberg ME, Fu H (2001) 14-3-3 inhibits Bad-induced cell death through interaction with serine-136. Mol Pharmacol 60:1325-1331.

Mattson MP (2000) Apoptosis in neurodegenerative disorders. Nat Rev Mol Cell Biol 1:120-129.

Moriwaki A, Lu YF, Tomizawa K, Matsui H (1998) An immunosuppressant, FK506, protects against neuronal dysfunction and death but has no effect on electrographic and behavioral activities induced by systemic kainate. Neuroscience 86:855-865.

Noshita N, Lewen A, Sugawara T, Chan PH (2001) Evidence of phos- phorylation of Akt and neuronal survival after transient focal cerebral ischemia in mice. J Cereb Blood Flow Metab 21:1442-1450.

Ouyang YB, Tan Y, Comb M, Liu CL, Martone ME, Siesjo BK, Hu BR (1999) Survival- and death-promoting events after transient cerebral ischemia: phosphorylation of $\mathrm{Akt}$, release of cytochrome $\mathrm{C}$ and Activation of caspase-like proteases. J Cereb Blood Flow Metab 19:1126-1135

Paxinos P, Watson C (1997) The rat brain in stereotaxic coordinates, Ed 3. London: Academic.

Peng CY, Graves PR, Thoma RS, Wu Z, Shaw AS, Piwnica-Worms H (1997) Mitotic and G2 checkpoint control: regulation of 14-3-3 protein binding by phosphorylation of $\mathrm{Cdc} 25 \mathrm{C}$ on serine-216. Science 277:1501-1505.

Pollard H, Charriaut-Marlangue C, Cantagrel S, Represa A, Robain O, Moreau J, Ben-Ari Y (1994) Kainate-induced apoptotic cell death in hippocampal neurons. Neuroscience 63:7-18.

Reed JC (1998) Bcl-2 family proteins. Oncogene 17:3225-3236.

Reibel S, Depaulis A, Larmet Y (2001) BDNF and epilepsy-the bad could turn out to be good. Trends Neurosci 24:318-319.

Sakhi S, Sun N, Wing LL, Mehta P, Schreiber SS (1996) Nuclear accumulation of p53 protein following kainic acid-induced seizures. NeuroReport 7:493-496.

Sloviter RS, Dean E, Sollas AL, Goodman JH (1996) Apoptosis and necrosis induced in different hippocampal neuron populations by repetitive perforant path stimulation in the rat. $\mathrm{J}$ Comp Neurol 366:516-533.

Snyder SH, Lai MM, Burnett PE (1998) Immunophilins in the nervous system. Neuron 21:283-294.

Springer JE, Azbill RD, Nottingham SA, Kennedy SE (2000) Calcineurin-mediated BAD dephosphorylation activates the caspase-3 apoptotic cascade in traumatic spinal cord injury. $\mathrm{J}$ Neurosci 20:7246-7251.

Subramanian RR, Masters SC, Zhang H, Fu H (2001) Functional conservation of 14-3-3 isoforms in inhibiting bad-induced apoptosis. Exp Cell Res 271:142-151.

Sun YF, Yu LY, Saarma M, Timmusk T, Arumae U (2001) Neuronspecific Bcl-2 homology 3 domain-only splice variant of Bak is antiapoptotic in neurons, but pro-apoptotic in non-neuronal cells. J Biol Chem 276:16240-16247.

Tan Y, Demeter MR, Ruan H, Comb MJ (2000) BAD Ser-155 phosphorylation regulates $\mathrm{BAD} / \mathrm{Bcl}-\mathrm{XL}$ interaction and cell survival. J Biol Chem 275:25865-25869.

Todd A, Cossons N, Aitken A, Price GB, Zannis-Hadjopoulos M (1998) Human cruciform binding protein belongs to the 14-3-3 family. Biochemistry 37:14317-14325.

Tuunanen J, Lukasiuk K, Halonen T, Pitkanen A (1999) Status epilepticus-induced neuronal damage in the rat amygdaloid complex: distribution, time-course and mechanisms. Neuroscience 94:473-495.

Viswanath V, Wu Z, Fonck C, Wei O, Boonplueang R, Andersen JK (2000) Transgenic mice neuronally expressing baculoviral p35 are resistant to diverse types of induced apoptosis, including seizureassociated neurodegeneration. Proc Natl Acad Sci USA 97:2270-2275.

von Ahsen O, Renken C, Perkins G, Kluck RM, Bossy-Wetzel E, Newmeyer DD (2000) Preservation of mitochondrial structure and function after Bid- or Bax-mediated cytochrome c release. J Cell Biol 150:1027-1036.

Wang HG, Pathan N, Ethell IM, Krajewski S, Yamaguchi Y, Shibasaki F, McKeon F, Bobo T, Franke TF, Reed JC (1999) $\mathrm{Ca}^{2+}$-induced apoptosis through calcineurin dephosphorylation of BAD. Science 284:339-343

Waterman MJ, Stavridi ES, Waterman JL, Halazonetis TD (1998) ATM-dependent activation of p53 involves dephosphorylation and association with 14-3-3 proteins. Nat Genet 19:175-178.

Wei MC, Zong WX, Cheng EH, Lindsten T, Panoutsakopoulou V, Ross AJ, Roth KA, MacGregor GR, Thompson CB, Korsmeyer SJ (2001) Proapoptotic BAX and BAK: a requisite gateway to mitochondrial dysfunction and death. Science 292:727-730.

Yang E, Zha J, Jockel J, Boise LH, Thompson CB, Korsmeyer SJ (1995) $\mathrm{Bad}$, a heterodimeric partner for Bcl-XL and Bcl-2, displaces Bax and promotes cell death. Cell 80:285-291.

Yano S, Morioka M, Fukunaga K, Kawano T, Hara T, Kai Y, Hamada J, Miyamoto E, Ushio Y (2001) Activation of Akt/protein kinase B contributes to induction of ischemic tolerance in the CA1 subfield of gerbil hippocampus. J Cereb Blood Flow Metab 21:351-360.

Zha J, Harada H, Yang E, Jockel J, Korsmeyer SJ (1996) Serine phosphorylation of death agonist BAD in response to survival factor results in binding to 14-3-3 not BCL-X(L). Cell 87:619-628.

Zilliacus J, Holter E, Wakui H, Tazawa H, Treuter E, Gustafsson JA (2001) Regulation of glucocorticoid receptor activity by 14-3-3 dependent intracellular relocalization of the corepressor RIP140. Mol Endocrinol 15:501-511.

Zou H, Henzel WJ, Liu X, Lutschg A, Wang X (1997) Apaf-1, a human protein homologous to $C$. elegans $\mathrm{CED}-4$, participates in cytochrome c-dependent activation of caspase-3. Cell 90:405-413. 